\title{
An Analysis and Simulation of Cellular Automaton
}

\author{
Zhijie Li, Xiaodong Duan ${ }^{+}$, Cunrui Wang, Jian Yun \\ ${ }^{1}$ School of Computer Science \& Engineering, Dalian Nationalities University, Dalian Liaoning \\ 116600, China \\ ${ }^{2}$ Dalian Key Lab of Digital Technology for National Culture, Dalian Nationalities University, Dalian \\ Liaoning 116600, China \\ ${ }^{+}$Corresponding author \\ email: minzudigital@dlnu.edu.cn
}

Keywords: Cellular Automaton; Digital Life; Evolution; Stability

\begin{abstract}
The life evolutionary model is researched and simulated as well as other typical cellular automaton model. Different consequences are simulated with various cell numbers, different initial states and different evolution rules. After results comparison among these methods, the factors impacting stability are analyzed. Results show that the complexity of cellular automaton can provide a simple and visual tool for the theory of complexity science.
\end{abstract}

\section{Introduction}

Cellular automaton (CA) is an important tool and theoretical method for researching artificial life. There are many important achievements in the research of artificial life. For example, the cellular automaton model proposed by von Neumann [1]; the automaton components that constitute a self-reproduction given by Codd E F [2]; and the most famous "life" game program compiled by the John Conwey of the University of Cambridge [3,4].

As an ideal mathematical model for studying complex system, the thinking of the cellular automaton is: Let computer calculate iteratively using extremely simple algorithms, then you can get the very complex model gradually, and it can be used to explain all the natural phenomena [5].

Cellular automaton [6-8] is a dynamic system with discrete time and space. The evolution of dynamic system is composed of a large number of cells through a simple interaction. Different from the general dynamic model, the cellular automaton is not determined by a strict definition of a physical equation or a function, but rather a series of the rules constructed by models. The model that satisfies these rules can be regarded as an automaton model. Its characteristics are that the time, space and state are all discrete; each variable is only limited to a limited number of states; and the rules of state changing in time and space are local. The cellular automaton can be used to study a lot of general phenomena, including communication, information transmission, calculation, structure, growth, reproduction, competition and evolution, etc. Therefore, the study of the cellular automaton is very important for the research of complex system, and also for the research of the large parallel computing theory. In this paper, we will compare 5 kinds of typical cellular automaton, analyze the behavior characteristics and the stability of the model, and hence provide the reference for the complex theory.

\section{The composition of the cellular automaton}

The cellular automaton is composed of four parts, including cellular, cellular space, neighbor and rule four parts.

Cell is also known as the unit, the base element, is the most basic element of the cellular automaton. Cell scattered in the lattice of discrete one-dimensional, two-dimensional or multidimensional Euclidean space. They have their own status. The set of all possible states of each cell is called cellular state set, which is generally expressed as the discrete set of integers $\left\{\mathrm{s}_{0}\right.$,

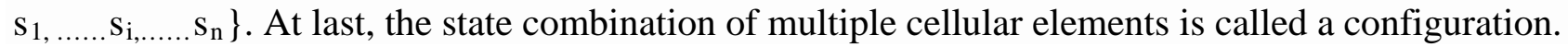


Cellular space is a collection of spatial nodes on which cell distributed. For the space division of the cellular space, it can be arbitrary dimension Euclidean space in theory. Currently, most of the studies focused on one-dimensional and two-dimensional cellular automaton. For the one-dimensional cellular automaton, cells distribute with equal interval on a straight line, which infinitely extended to both sides. But for the high dimensional cellular automaton, the division rule may have a variety of forms. For example, the most common two-dimensional cellular automaton, the space division is shown in Fig. 1.

Neighbor is usually determined by radius. That means the elements within a radius of a cell are all the neighbors of the cell. The neighbor definition of two-dimensional cellular automaton is more complex. We select the most common rules of the square grid division, which usually have the following forms, see Fig.1. The black element in the picture represents the central element of the cell, and the gray cells represent the neighbor of the black central cell. So, the eight cells includes the upper, lower, left, right, upper left, lower left, upper right, lower right, next to the center cell are the neighbors.

Rule is a dynamic function to determine the next cellular state according to the current state of a cell and its neighbors. For the sake of simplicity, the rule is a state transfer function. We call all the possible states of a cell, together with the rules for deciding the state of the cell, the transformation function. This function constructs a simple, discrete space and time. It can be written as $\mathrm{f}: \mathrm{s}_{\mathrm{i}}{ }^{\mathrm{t}}{ }^{+1}=\left(\mathrm{s}_{\mathrm{i}}{ }^{\mathrm{t}}\right.$, $\mathrm{s}_{\mathrm{n}}{ }^{\mathrm{t}}$ ), $\mathrm{s}_{\mathrm{n}}{ }^{\mathrm{t}}$ is the neighbor state combination of time T. We call $\mathrm{f}$ the local mapping or local rules for the cellular automaton.

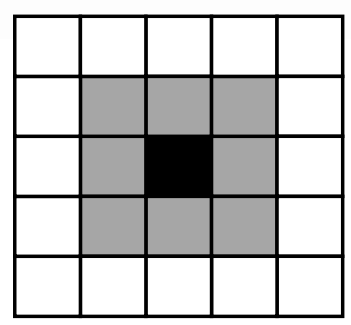

Fig. 1. Common division of cellular space and neighbor

\section{The evolution models with different rules}

Five cellular automaton models will be analyzed in this section. First, we discuss the life evolution model. In this model, the rules suppose that there is a square grid, in which a life cell is put in. There are only two states in each cell, that is "life" or "death". In programming, we use the two-dimensional $n \times n$ matrix to represent cellular space. Each element represents a cell. The value 1 means the cell is living. In contrast, the value 0 means the cell is dead. In the $t+1$ time step, the cellular state is determined by the its own state and surrounding neighbor state in the time step. Its rules are as follows:

(1) the distribution of the cells is in a uniform grid;

(2) the cell has two states, 0 and 1 , and 0 represents "death", and the 1 represents "living";

(3) the neighbors are the 8 cells next to the central cell;

(4) the state of a cell is determined by the state of its own and the 8 neighbors;

(5) the evolution rule: at the current time, if there are three living cells around a cell, the cell is living. Namely, if the cell is dead originally, then it is living now; otherwise remains unchanged. If there are two living cells around a cell, the state of the cell remains unchanged. In other cases, the cell is dead. Namely, if the cell is living originally, then it dies; otherwise remains unchanged.

The evolution rule appears simple, but it reflects the survival law in nature. That is to say, if the lives of the same kind are too few, the cell will die without enough help. If the lives of the same kind are too much, the cell will also die because of the lack of enough resources caused by competition. Since the evolution process will last a long time, we only select two time steps to display the space state shown by the cells, shown in Fig. 2. 


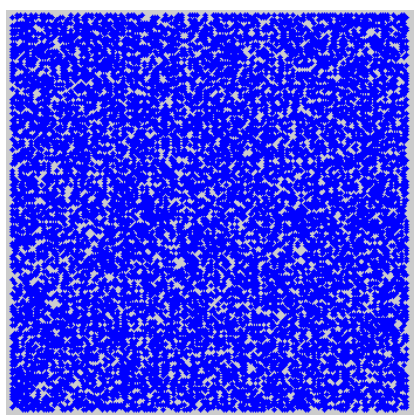

(a) the state at 0 iteration

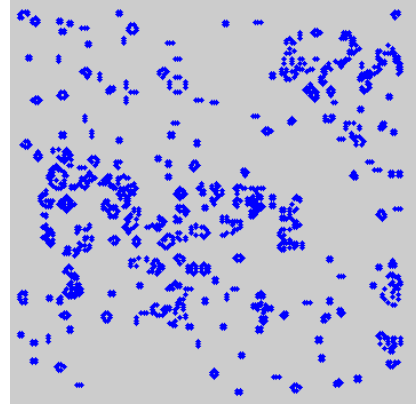

(b) the state at 300 iteration

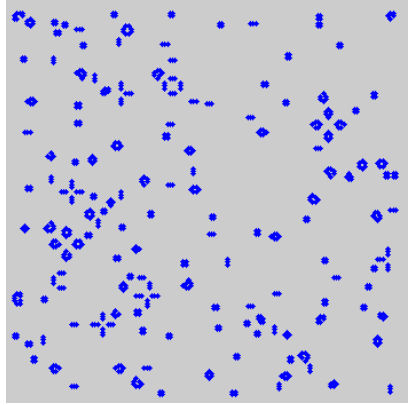

(c) the state at 1000 iteration

Fig. 2 The cellular space state of the life evolution model

For reason of the limited space, we only discuss the life evolution model in detail. The other four cellular automaton models are summarized in the following table 1.

Table 1. Evolutionary rules of different cellular automaton

\begin{tabular}{|c|c|c|c|}
\hline & Rule 1 & Rule 2 & Rule 3 \\
\hline $\begin{array}{c}\text { Surface } \\
\text { Tension } \\
\text { model }\end{array}$ & $\begin{array}{c}\text { Sum the } 8 \text { nearest } \\
\text { neighbors and the } \\
\text { cell itself. }\end{array}$ & $\begin{array}{c}\text { If the sum }<4 \text { or sum }=5 \text { then } \\
\text { the state }=0 \text {. }\end{array}$ & Otherwise the state $=1$. \\
\hline $\begin{array}{c}\text { Excitable } \\
\text { media model }\end{array}$ & $\begin{array}{l}\text { Cells can be in } 10 \\
\text { different states. } \\
\text { State } 0 \text { is resting. } \\
\text { States } 1-5 \text { are } \\
\text { active, and states } \\
6-9 \text { are refractory. }\end{array}$ & $\begin{array}{c}\text { Count the } 8 \text { nearest neighbors } \\
\text { of each cell which are in one } \\
\text { of the active states. If the sum } \\
\text { is greater or equal to } 3 \text { (at } \\
\text { least three active neighbors) } \\
\text { then cell=1. }\end{array}$ & $\begin{array}{l}\text { States } 1 \text { to } 9 \text { occur stepwise with no more } \\
\text { input. If state }=1 \text { then the next state }=2 \text {. If } \\
\text { state }=2 \text { then the next state }=3 \text {, and similarly } \\
\text { of all the states up to } 9 \text {. If state }=9 \text { then the } \\
\text { next state }=0 \text { and the cell is back at rest. }\end{array}$ \\
\hline $\begin{array}{l}\text { Diffusion } \\
\text { limited } \\
\text { aggregation }\end{array}$ & $\begin{array}{l}\text { Particles are } \\
\text { assumed to be } \\
\text { bouncing around } \\
\text { in some dense } \\
\text { (but invisible) } \\
\text { liquid. }\end{array}$ & $\begin{array}{l}\text { At every time step, rotate the } \\
4 \text { cells either clockwise or } \\
\text { counterclockwise by one cell } \\
\text { with equal probability. The } \\
\text { rotation randomizes the } \\
\text { velocities. }\end{array}$ & $\begin{array}{l}\text { After the move, if one or more of the eight } \\
\text { nearest neighbor is a fixed, sticky particle, } \\
\text { then freeze the particle and make it sticky }\end{array}$ \\
\hline $\begin{array}{l}\text { Forest Fire } \\
\text { model }\end{array}$ & $\begin{array}{l}\text { Cells can be in } 3 \\
\text { different states: } \\
\text { state }=0 \text { is empty, } \\
\text { state }=1 \text { is burning } \\
\text { and state }=2 \text { is } \\
\text { forest }\end{array}$ & $\begin{array}{l}\text { If one or more of the } 4 \\
\text { neighbors if a cell is burning } \\
\text { and it is forest (state=2) then } \\
\text { the new state is burning } \\
\text { (state }=1 \text { ). }\end{array}$ & $\begin{array}{l}\text { There is a low probability of a forest cell } \\
\text { (state }=2 \text { ) starting to burn on its own. A cell } \\
\text { which is burning (state }=1 \text { ) becomes empty } \\
\text { (state }=0 \text { ). There is a low probability of an } \\
\text { empty cell becoming forest to simulate } \\
\text { growth. The fire which burns to left side } \\
\text { will start fires on the right. The top and } \\
\text { bottom are similarly connected. }\end{array}$ \\
\hline
\end{tabular}

\section{Stability analysis}

In theory, the evolutionary space of cellular automaton is usually unlimited in in each dimension, which is good for reasoning and study in theory. However, in reality, it is impossible to realize the ideal condition on the computer. Therefore, it is needed to define different boundary conditions. There are three main types: periodic, reflection and constant value. All the above models in table 1 are designed under the condition of constant value, and with eight neighbors and two states. After evolving to certain iteration, if the cell number with state ' 1 ' tends to be stable, then the cellular automaton belongs to stable; if the cell number with state ' 1 ' changing periodically, then the cellular automaton belongs to periodic; if there is no obvious regularity, then the cellular automaton belongs to complex. This classification method is based on the statistical properties of the one-dimensional cellular evolution process.

During the simulations, we can observe the cell number with state ' 1 ' ' in the evolution process of various cellular automata. From the results, it can be concluded that the life evolution model, surface tension model, diffusion limited aggregation model belong to stable type, because they all 
get a stable pattern finally. The excitable media model belongs to periodic, since the pattern changing periodically. The forest fire model belongs to complex, because that the pattern changing unlimitedly without any regularity or periodicity. The cell number of the three main types of cellular automaton is shown in Fig. 3.

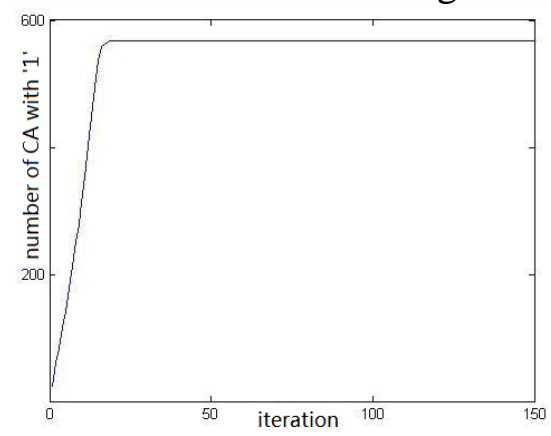

(a)Stable cellular automaton

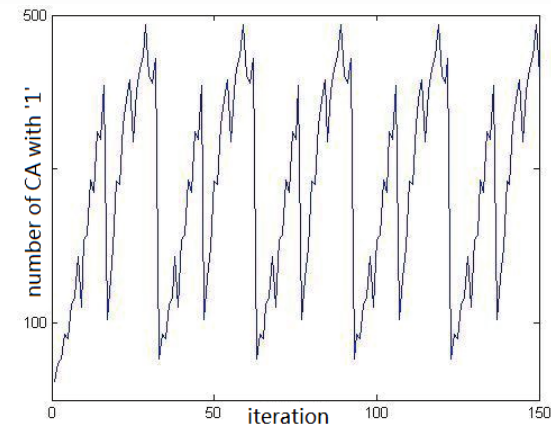

(b)Periodic cellular automaton

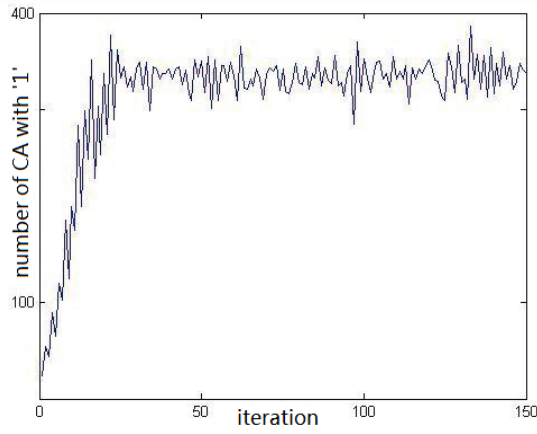

(c)complex cellular automaton

Fig. 3. Statistics of the cell number with state "1" in different cellular automaton

\section{Conclusion}

This paper studied the theory of cellular automata from the viewpoint of computer. The experiments run on MATLAB are made to study characteristics of various types of cellular automata. Results show that the stability of cellular automata is depended on the evolutionary condition, and different factors such as cell number, initial state, and boundary rule can affect the stability also. The conclusion indicates that the cellular automata can provide reference for the complex theoretical derivation and natural phenomena.

\section{Acknowledgement}

This work is supported by the National Natural Science Foundation of China under Grant No. 61370146, No. 61040054, and the Fundamental Research Funds for the Central Universities under Grant (No.DC201502030203, No.DC201502030403) and the Humanity and Social Science Foundation of Ministry of Education of China No. 12YJCZH263.

\section{References}

[1] J von Neumann. Theory of Self-Reproducing Automata [M]. Urbana: University of Illionois, 1966.

[2] Codd E F. Cellular Automata [M]. New York: Academic Press, 1968.

[3] John Conway. Game of Life [J]. Scientific American, 1970, 223: 120 123.

[4] Gradner M. The Fantastic Combinations of John Conway' s New Solitaire Game Life [J]. Scientific American, 1970, 223(4): 120 123.

[5] Wolfram S, A New Kind of Science [M]. Wolfram Media Inc, 2002.

[6] Wolfram S. Cellular Automata as Models of Complexity [J]. Nature, 1984, 311(6): 419 424.

[7] F. Peper, J. Lee, T. Isokawa. Fault-Tolerance in Nanocomputers: A Cellular Array Approach [J]. IEEE Transactions on Nanotechnology, 2004, 3(1): 187 201.

[8] Rmentrout B E, Eshet E. Cellular Automata Approaches to Biological Modeling [J]. Journal of Theoretical Biolog 1993, 160: 97 133. 